CIENCIA 8(2), 168-180, 2000

Maracaibo, Venezuela

\title{
Corrientes superficiales en la Bahía de Bergantín (Estado Anzoátegui, Venezuela)
}

\author{
Julián J. Castañeda*, Rubén Aparicio Castro y William J. Senior \\ Dpto. de Oceanografia, Instituto Oceanográfico de Venezuela, Universidad de Oriente, Venezuela \\ Recibido: 18-10-98. Aceptado: 14-12-99
}

\section{Resumen}

Un conjunto de observaciones de corrientes superficiales realizadas por el Instituto Oceanográfico de Venezuela en la Bahía de Bergantín, se obtuvieron durante algunos meses de 1989. Ese conjunto de mediciones se analiza a la luz de nuevas metodologías; en particular se describe y analiza la naturaleza del registro de corriente y su posible vinculación con agentes forzantes. Se encuentra que: a) Opuesto a la creencia generalizada de que la bahía de Bergantín es el principal generador de impactos sobre la bahía de Pozuelos, en virtud de las operaciones propias de la industria petrolera que allí se desarrollan, se demuestra que aguas superficiales dentro del rango de 3 kilómetros, ingresan desde Pozuelos a Bergantín, bajo condiciones de vientos débiles y mareas llenantes. b) Espectralmente la banda de frecuencia asociada a vientos del NE con períodos de 6, 12 y 24 horas es el agente con mayor potencial inductor de corrientes superficiales. La coincidencia de esas bandas energéticas con la de los constituyentes cuarto, semidiurnos y diurnos de la marea, representa un factor de alto riesgo para las operaciones de los tanqueros petroleros dentro y fuera de la bahía. De particular interés resulta ser la descripción de la dinámica, tanto de corrientes marinas como del viento observada durante el mes de septiembre, caracterizada principalmente por un período de reversión del régimen de vientos locales que afectaron la zona de estudio soplando desde el SUR. Esta reversión genera un cambio sustancial el patrón de circulación superficial, haciendo que por períodos mayores de 46 horas, aguas provenientes de Pozuelos ingresen a la Bahía de Bergantín. Se concluye estableciendo como necesidad primordial, la vigilancia permanente de la variable intercambio de agua entre la Bahía de Bergantín y sus áreas adyacentes ( a través de registros sinópticos de los parámetros vientos, mareas y corrientes). Ello potenciará los esquemas de contingencia existentes por parte de la industria petrolera nacional para esta localidad.

Palabras clave: Análisis espectral; contaminación costera; corrientes.

\section{Surface currents in the Bay of Bergantín (Anzoategui State, Venezuela)}

\begin{abstract}
A set of measurements of sea surface currents obtained by the Instituto Oceanográfico de Venezuela in Bergantín Bay during various months in 1989 are re-analysed with new approaches. In particular the source of the current records is described and their likely connection with forcing agents is analysed. The following results are found. 1) Contrary to the longstanding belief that Bergantín Bay is the main source of pollution of Pozuelos Bay (owing to the

* Autor para la correspondencia. E-mail: jjcasta@sucre.udo.edu.ve
\end{abstract}


oil related activities that take place there), it is shown that the opposite effect may occur under conditions of weak winds and flood tides; i.e., surface water in Pozuelos Bay within a range of about $3 \mathrm{~km}$ has the potential to enter into Bergantín Bay. 2) Spectral analysis of the wind signal showed that winds from the NE with periods of 6,12 and 24 hours are the most energetic. The occurrence of these events in coincidence with tidal quarter-, semi- and diurnal components represents a high risk factor for oil tanker operations in both Bergantín Bay and Pozuelos Bay. Of particular interest was the description of the dynamic, both of the sea surface current and the wind during September, at which time the wind field showed a reversal of direction, blowing mainly from the south. This reversal creates a dramatic change in the local surface circulation, allowing sea surface waters from Pozuelos Bay to penetrate into Bergantín Bay for periods longer that 46 hours. It is concluded that it is of primary importance to establish as a primary goal the observation of the variable water exchange between Bergantin Bay and its neighbouring waters (by mean of the sampling of synoptic records of wind, sea surface levels and currents). Such measurements will help our national oil industry to improve the quality of the existent plans to control oils spills in the area.

Key words: Coastal pollution; currents; spectral analysis.

\section{Introducción}

Como país productor y exportador de hidrocarburos, Venezuela utiliza sus costas en el Mar Caribe para el asiento de macroestructuras destinadas a una variada e intensa actividad relacionada, principalmente, con el refinamiento y transporte de crudos y sus derivados. En consecuencia, su margen litoral y cuerpo de aguas costeros precisan de políticas de resguardo ambiental, en la cual la dispersión y transporte de contaminantes en el medio marino juega un papel crítico. En este sentido, tanto la necesidad de identificar las fuentes de contaminación (vertidos, efluentes, microderrames, etc.), como la de caracterizar el patrón de circulación en el cuerpo de agua pertinente, exigen idéntica atención a la hora de planificar programas de contingencia y de establecer las responsabilidades debidas.

En este trabajo se utiliza un grupo de escasos registros correntométricos conocidos de la zona para analizar la variabilidad temporal del flujo superficial en una bahía ocupada por la industria petrolera nacional y se discute su incidencia sobre potenciales planes de contingencia a ser diseñados para minimizar los posibles efectos negativos am- bientales ocasionados por eventuales derrames y vertidos de substancias contaminantes en el medio marino.

\section{Descripción de la Zona de Estudio}

La Bahía de Bergantín (Figura 1) es una bahía semicerrada ubicada sobre la porción oriental del margen costero del Estado Anzoátegui, ocupando una extensión espacial cercana a los $5 \mathrm{~km}^{2}$, demarcada por el rango longitudinal $64^{\circ} 36^{\prime} 30^{\prime \prime}$ y $64^{\circ} 38^{\prime}$ 00" O, con su eje zonal ubicado a la latitud $10^{\circ} 15^{\prime} \mathrm{N}$. La bahía ocupa un volumen aproximado de $4 \times 10^{7} \mathrm{~m}^{3}$ y se conecta hacia el O (Paso Oeste) con la Bahía de Pozuelo y hacia el N (Paso Norte) con el corredor marino constituido por la línea de costa continental, el cual a su vez, se conecta hacia el Este con la cercana Bahía de Pertigalete. La batimetría de la bahía revela máximas profundidades en sus pasos, producto de las periódicas operaciones de dragado a que son sometidos estos canales de acceso de la bahía.

Esta bahía se encuentra en un margen litoral caracterizado por presentar una extensiva ocupación industrial, resaltando la presencia de infraestructuras dedicadas a uno de los más importantes terminales na- 


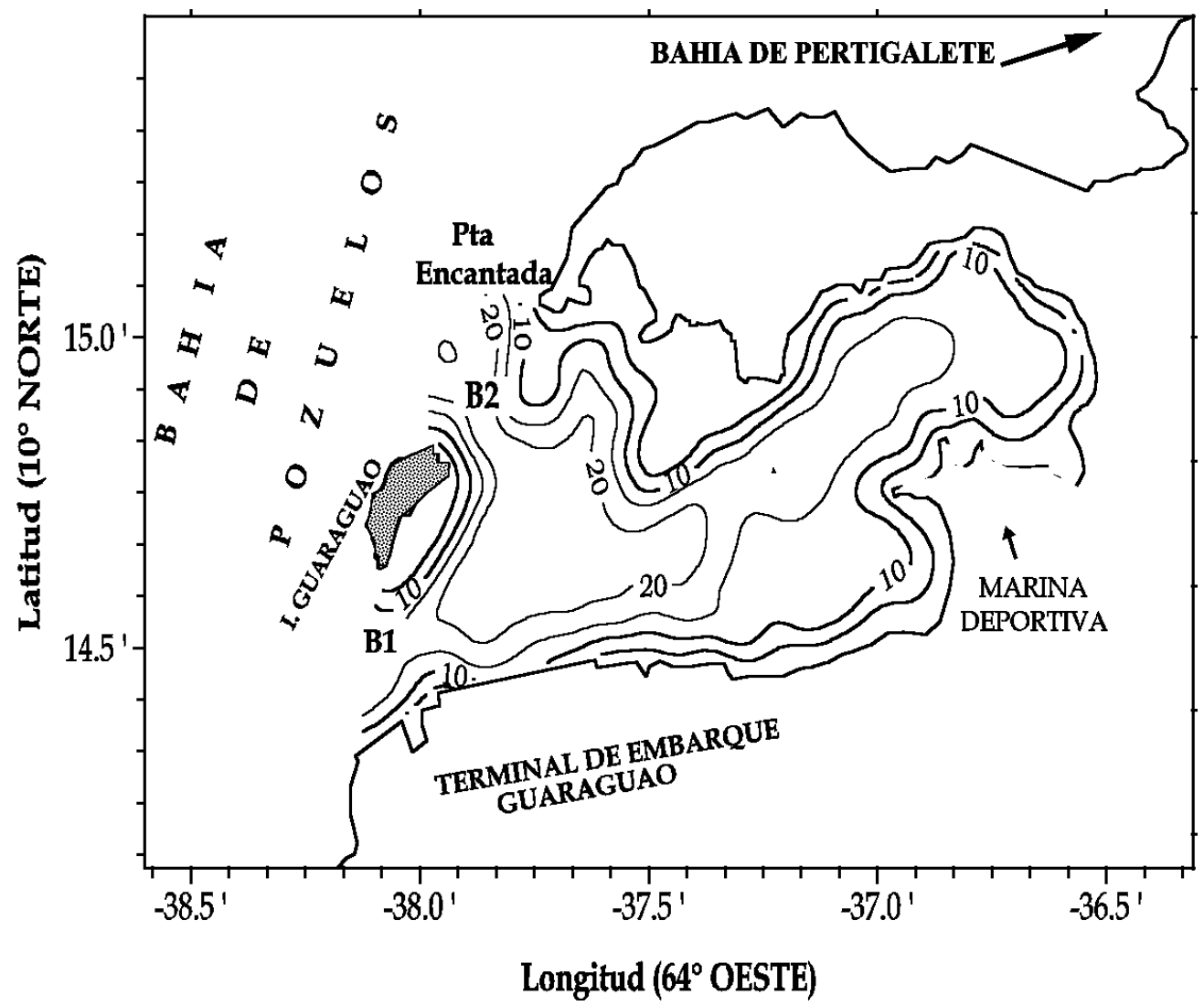

Figura 1. Ubicación geográfica del área de estudio y localización de las estaciones correntométricas. Las coordenadas geográficas están expresadas en décimas de minutos. Las isobatas se expresan en metros.

cionales de hidrocarburos y derivados (Terminal de Embarque Guaraguao), además de la Refinería El Chaure, en el extremo oriental de la bahía, junto a una laguna de lastre y una marina de lanchas deportivas. Todo ello implica un cuantioso y frecuente tráfico marítimo que involucra tanto las actividades turísticas y pesqueras como las propias de la industria petrolera.

\section{Metodología y Descripción de las Medidas}

En la Figura 1 se presentan las posiciones de las estaciones correntométricas usadas en este estudio. Un resumen de las estadísticas básicas de esas mediciones, se presenta en la Tabla 1. La estación denominada $\mathrm{B} 1$ se ubica en el paso de aguas entre la $\mathrm{Ba}-$ hía de Bergantín y la de Pozuelos, específicamente entre Isla Guaraguao y el terminal de embarque de hidrocarburos. La estación B2, se ubica en el paso norte de la Bahía de Bergantín, especificamente entre Isla Guaraguao y Punta Encantada. Las mediciones se realizaron con un correntímetro Doppler marca Neil Brown, con resolución de 1 $\mathrm{cm} / \mathrm{s}$. Todas las mediciones se realizaron aproximadamente a un metro de la superficie. Los registros consistieron en mediciones promediadas a intervalos de $5 \mathrm{~min}$. La extensión de las mediciones varió entre $18 \mathrm{y}$ $26 \mathrm{~h}$ consecutivas. Las observaciones se convirtieron en coordenadas cartesianas coincidiendo el eje de las abscisas con la línea de costa. Es de resaltar que a pesar de la importancia industrial de la zona de estudio, no se conocen investigaciones previas 
Tabla 1

Estadísticas básicas de las series de tiempo de las mediciones de corrientes en la Bahía de Bergantín

\begin{tabular}{|c|c|c|c|c|c|}
\hline & \multicolumn{5}{|c|}{ Estaciones } \\
\hline & $\mathrm{B} 1$ & $\mathrm{~B} 1$ & $\mathrm{~B} 1$ & $\mathrm{~B} 1$ & $\mathrm{~B} 2$ \\
\hline Fecha & $16 / 03 / 1989$ & $25 / 04 / 1989$ & 26/05/1989 & $05 / 09 / 1989$ & $26 / 04 / 1989$ \\
\hline Duración (h) & 19.42 & 24.75 & 24.25 & 46.42 & 20.75 \\
\hline $\begin{array}{c}\text { Intervalos de Rapidez } \\
(\mathrm{cm} / \mathrm{s})\end{array}$ & \multicolumn{5}{|c|}{ Frecuencia \% } \\
\hline $0-5$ & 24,03 & 17,51 & 29,55 & 0,54 & 6,83 \\
\hline $5-10$ & 52,79 & 35,35 & 47,42 & 33,57 & 26,51 \\
\hline $10-15$ & 22,75 & 30,64 & 15,81 & 49,91 & 52,61 \\
\hline $15-20$ & 0,43 & 8,08 & 4,12 & 15,08 & 12,45 \\
\hline $20-25$ & 0,00 & 5,72 & 3,09 & 0,72 & 1,61 \\
\hline $25-30$ & 0,00 & 2,69 & 0,00 & 0,00 & 0,00 \\
\hline $30-35$ & 0,00 & 0,00 & 0,00 & 0,18 & 0,00 \\
\hline $35-40$ & 0,00 & 0,00 & 0,00 & 0,00 & 0,00 \\
\hline $40-45$ & 0,00 & 0,00 & 0,00 & 0,00 & 0,00 \\
\hline $45-50$ & 0,00 & 0,00 & 0,00 & 0,00 & 0,00 \\
\hline Máx (cm/s) & 12,70 & 25,10 & 22,10 & 32,40 & 19,20 \\
\hline Min $(\mathrm{cm} / \mathrm{s})$ & 0,10 & 0,60 & 0,10 & 1,20 & 0,80 \\
\hline Media $(\mathrm{cm} / \mathrm{s})$ & 5,00 & 8,10 & 5,50 & 9,10 & 8,80 \\
\hline Desv. Estand. $(\mathrm{cm} / \mathrm{s})$ & 3,00 & 5,50 & 4,30 & 3,51 & 3,90 \\
\hline
\end{tabular}

Las direcciones están expresadas en grados Norte, B1 16/03/89 significa Bergantín, Estación 1, 16 Mar 1989.

que reporten mediciones correntométricas, de igual manera, no existe en la zona ni en la cercanía ningún registro de mareas operativo que puede ayudar a la obtención indirecta de los constituyentes mareales.

La información relativa a los vientos se obtuvo de la estación meteorológica más cercana ubicada en el Aeropuerto de Barcelona (alrededor de $10 \mathrm{~km}$ ), operada por las Fuerzas Aéreas de Venezuela. Los registros de vientos corresponden a mediciones horarias de magnitud y dirección, siguiendo la metodología estándar propuesta de la Organización Mundial de Meteorología, a la cual está adscrita la estación.
Las rutinas de análisis espectral de series de tiempo del software comercial MATLAB se usaron para el cálculo de la densidad de energía espectral de los registros de vientos y corrientes, siguiendo el método de Welch (1), con solapamiento a fin de suavizar y disminuir la varianza del espectro. En las gráficas de energías espectrales, las energías contenidas en las bandas de frecuencias, $f$, se representaron en decibeles y las bandas de frecuencia se sustituyeron por sus equivalentes en períodos, $T=1 / f$, en escalas logarítmicas de base 10 . Los espectros de potencia se obtuvieron a partir de la magnitud de las velocidades, tanto del viento como de las corrientes. 


\section{Resultados}

\section{Régimen de viento}

Es un hecho reconocido que el patrón de viento en la zona de estudio está controlado por los vientos alisios (2) que soplan persistentemente desde el Noreste. Sin embargo, a escala horaria el campo de viento presenta una marcada periodicidad cíclica. La Figura 2(a) presenta la distribución de las direcciones de donde soplan los vientos para el mes de marzo (época de mayor intensificación del régimen de vientos en toda la costa de Venezuela) en forma de histogramas. Esta distribución es muy similar a la observada para los meses de abril-julio. Claramente existe más de un $60 \%$ de ocurrencia de vientos que soplan en las bandas direccionales NE y NNE. En menor porcentaje de ocurrencia se registran vientos que soplan en la banda NNO.

La Figura 2(b) muestra la distribución temporal de la velocidad del viento. La dirección norte está representada por línea verticales orientadas hacia el tope de la página. $\mathrm{El}$ eje temporal se discrimina en horas sucesivas a partir de la medianoche del 15 de marzo. Para ayudar a la visualización se han introducido líneas verticales sucesivas y equidistantes que corresponden a la medianoche de cada día. Resalta la marcada periodicidad cíclica en la escala diurna, que se caracteriza por vientos débiles durante la primera parte del día y una intensificación de la magnitud que comienza alrededor del mediodia hasta la medianoche, alcanzando máximos cercanos a los $9 \mathrm{~m} / \mathrm{s}$, en horas de la tarde (15:00). La magnitud media de todo el registro es de $3,8 \mathrm{~m} / \mathrm{s}$.

Para completar la visión del régimen local de vientos, la Figura 2(c) presenta el resultado del análisis de la densidad de energía espectral aplicado a la serie temporal de la magnitud del viento, $W(t)$. En general la densidad espectral o potencia nos da información sobre el contenido energético de cada una de las señales presentes en el re- gistro de viento. La gráfica indica que la potencia del viento para eventos con períodos menores a $5 \mathrm{~h}$ es relativamente baja (alrededor de $5 \mathrm{db}$ ). Sin embargo, se observa que los eventos con períodos de $6 \mathrm{~h}(5 \mathrm{db}) 12 \mathrm{~h}$ (13 db) y $24 \mathrm{~h}(24 \mathrm{db})$ aportan la mayor cantidad de energía a la señal de viento. Ello no es más que una cuantificación de que el registro de viento podría intentar modelarse como una combinación de funciones trigonométricas con los períodos señalados. Esta descomposición de la energía del viento resulta de particular interés, desde el punto de vista de la dinámica local de la bahía, en vista de la correspondencia de esas bandas energéticas de frecuencias con las bandas energéticas de las mareas. En particular, las constituyentes quarto-, semi-y diurnas poseen constituyentes con períodos similares (3).

De particular interés resultó ser la distribución del patrón de vientos durante el mes de septiembre (Figura 3). Para este período se rompe el patrón de vientos dominantes del NorEste que se observa par el primer semestre del año y se imponen vientos que soplan desde la banda direccional Sur, con una frecuencia de ocurrencia del $73 \%$.

\section{Corrientes}

La Tabla 1 presenta un resumen de los parámetros estadísticos básicos de las series de tiempo de las corrientes superficiales en los dos puntos de observación.

En primer lugar, se observa que la rapidez de la corriente escasamente supera los $30 \mathrm{~cm} / \mathrm{s}$, siendo $32,4 \mathrm{~cm} / \mathrm{s}$ el máximo valor total encontrado en el muestreo de septiembre. La magnitud media de la corriente en los canales de la Bahía de Bergantín se puede tipificar por el valor medio de $4 \mathrm{~cm} / \mathrm{s}$.

La Figura 4 presenta una secuencia de gráficos de astillas para las series de tiempo de las corrientes superficiales medidas en las estaciones B1 y B2. Cada gráfica es representativa de una medición en un mes en particular. La magnitud del vector corriente está representada por la extensión de la as- 
a)

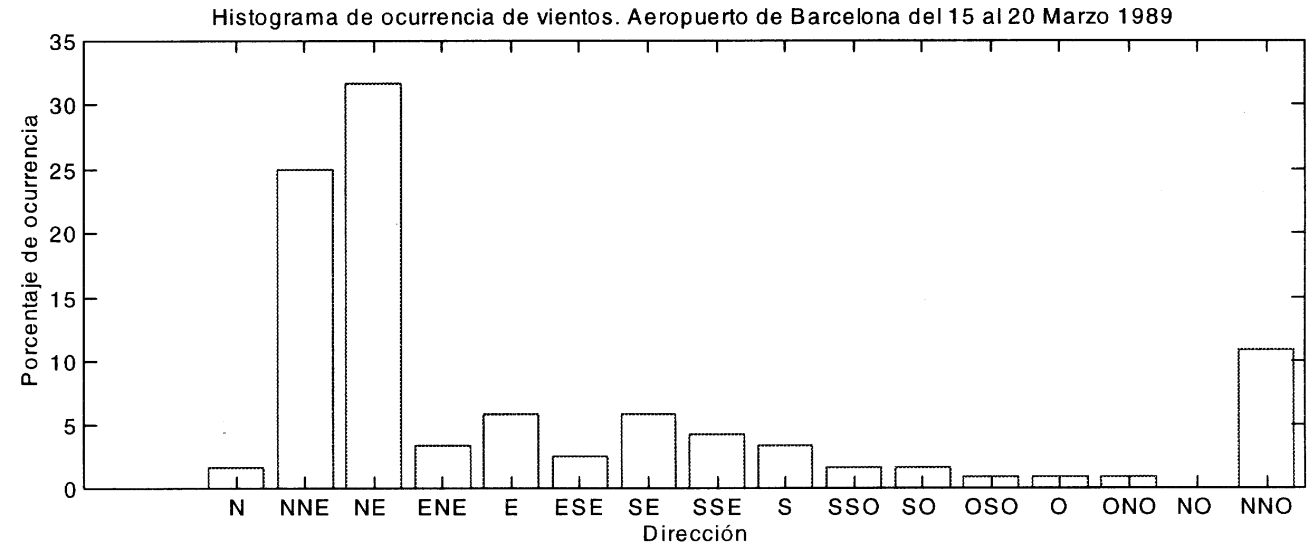

b)

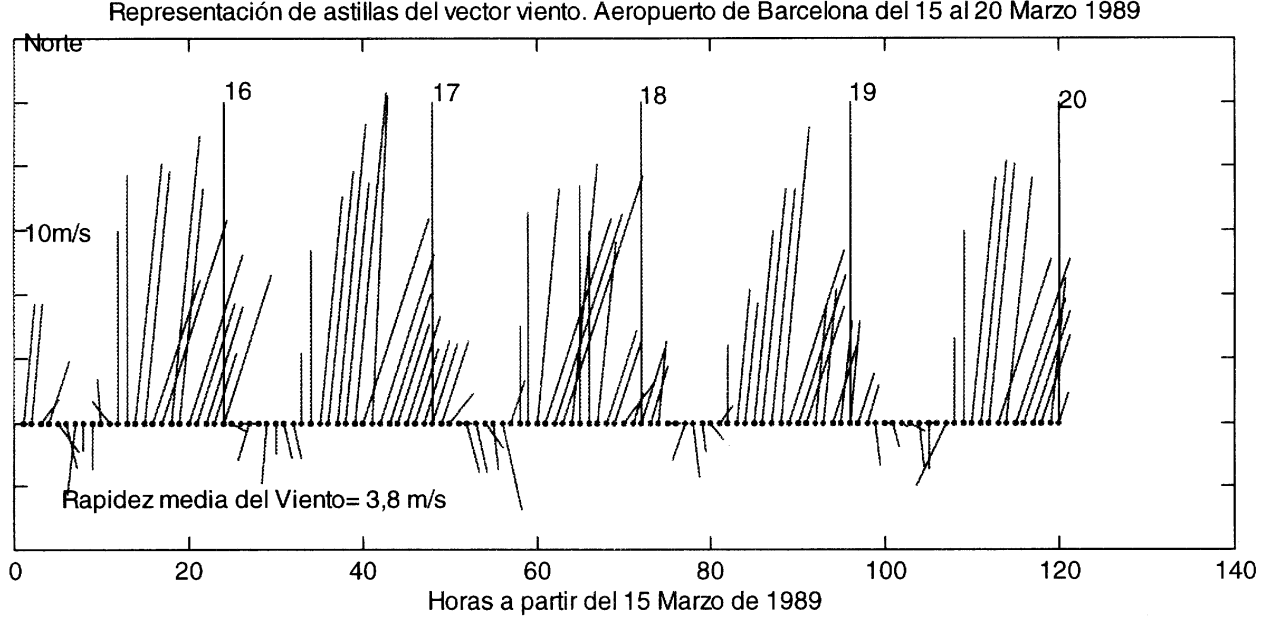

c)

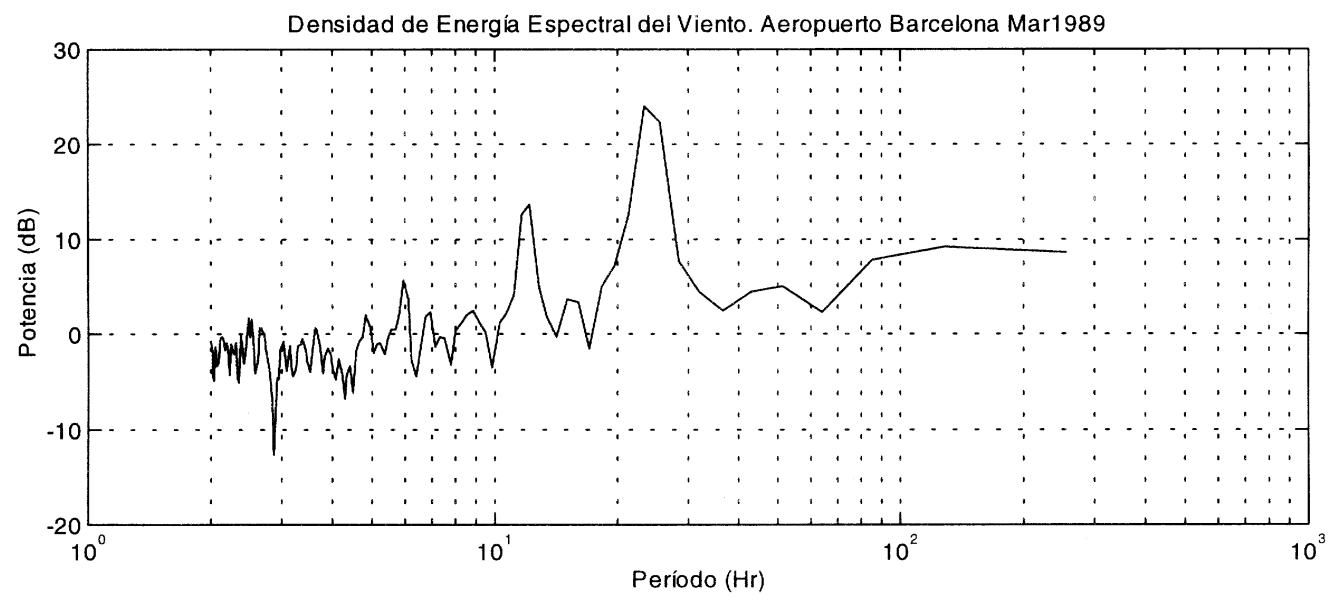

Figura 2. Composición gráfica de varias representaciones del vector velocidad del viento, para el período 15 al 20 de marzo de 1989, aeropuerto de Barcelona. a) Histograma de ocurrencia direccional del viento. b) Representación vectorial en forma de astillas del vector velocidad del viento; y c) Densidad de energía espectral o potencia de la magnitud del viento. 
(a)
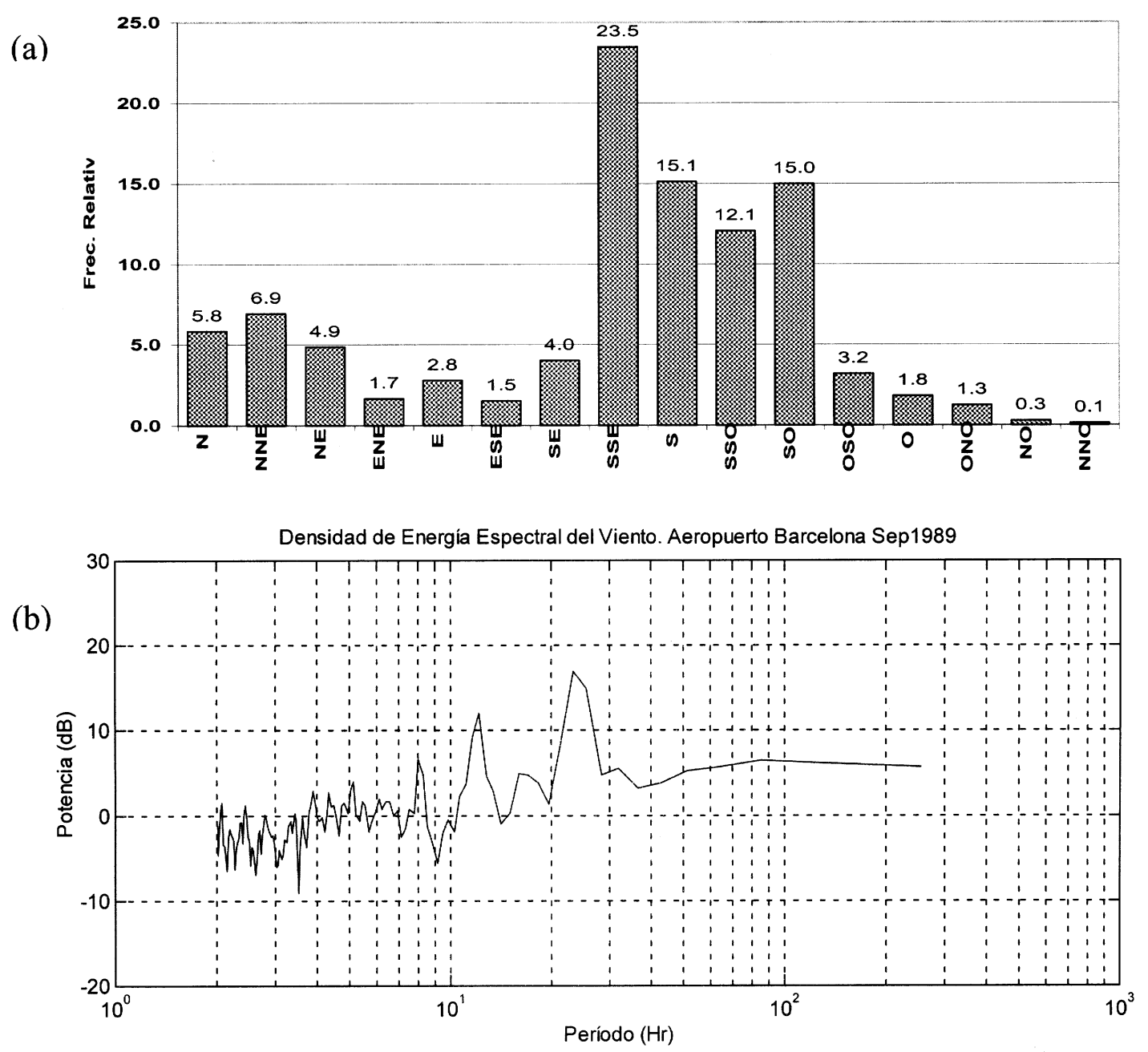

Figura 3. Varias representaciones del vector viento medido en Barcelona Septiembre de 1988. a) Histograma de frecuencias b) Densidad de energía espectral o potencia de la magnitud de la corriente.

tilla y su dirección y sentido por la inclinación y lugar en el plano al que señalan. A los fines de facilitar la interpretación visual, la dirección vertical en las gráficas se toma como referencia geográfica; Este en las primeras cuatro y Norte en la última gráfica La primera astilla de la serie representa la escala, que se explica por sí misma.

Durante el lapso de observación se encontró que la estación B2 presenta un flujo predominante hacia el Sur de la bahía. Ello parece indicar que a través de este canal, la bahía de Bergantín recibe el aporte de las aguas provenientes desde la adyacente $\mathrm{Ba}$ hía de Pertigalete (Figura 1), tal como fue establecido por Aparicio (4). Este típico flujo de canal posee una rapidez media aproximada de $9 \mathrm{~cm} / \mathrm{s}$. Nótese, que aunque existen períodos de corrientes casi nulas, la reversión de la corriente no sucede, a pesar de la reversión de flujo inducido por las mareas.

La estación $\mathrm{B} 1$ presenta un patrón más complejo. Son evidentes los flujos y reflujos de la corriente, típicos de un canal, induci- 
(a)

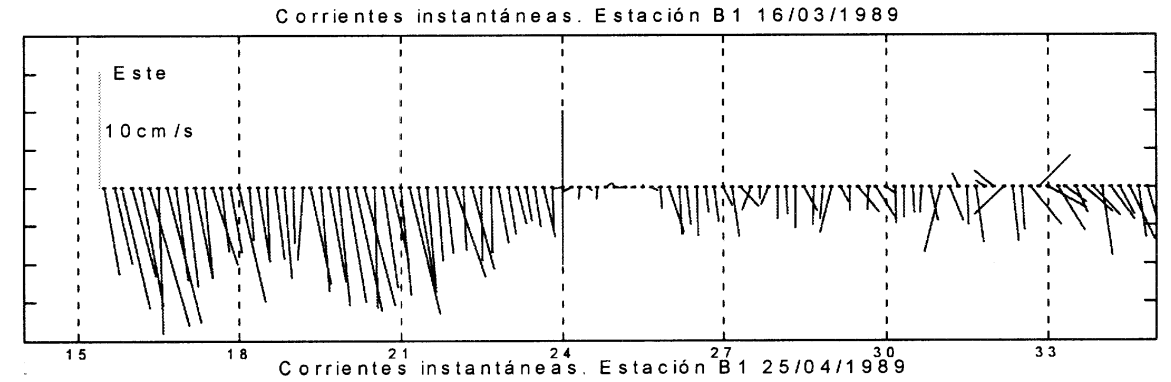

(b)

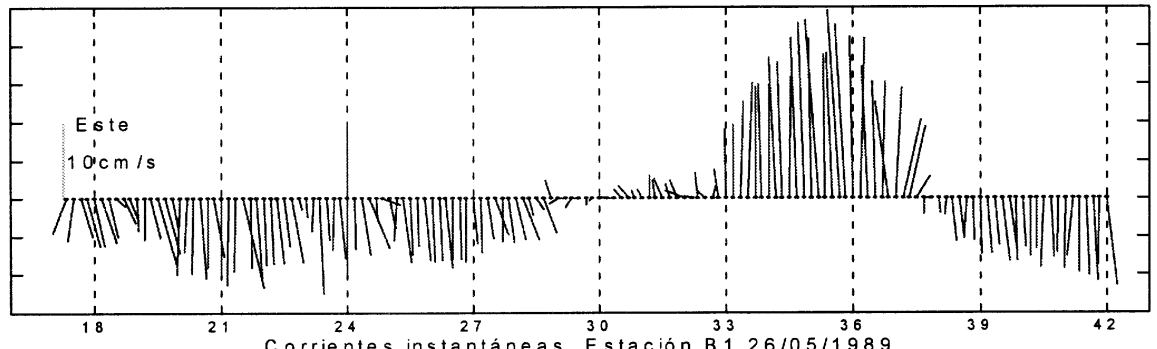

(c)

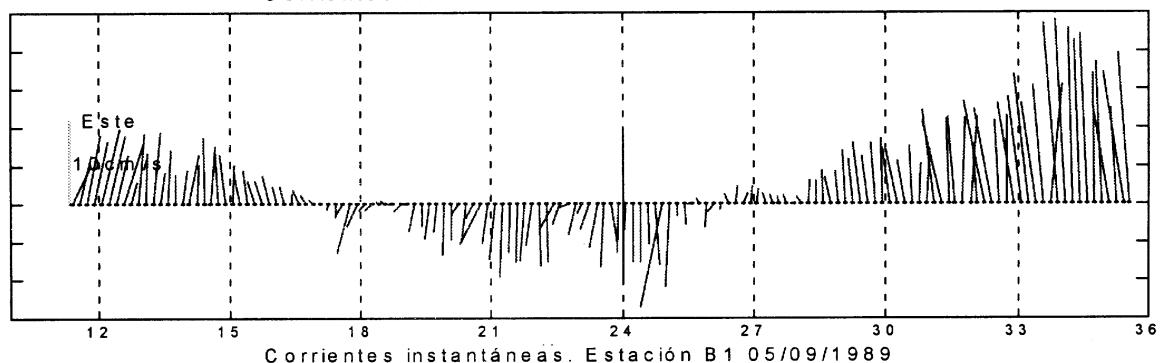

(d)

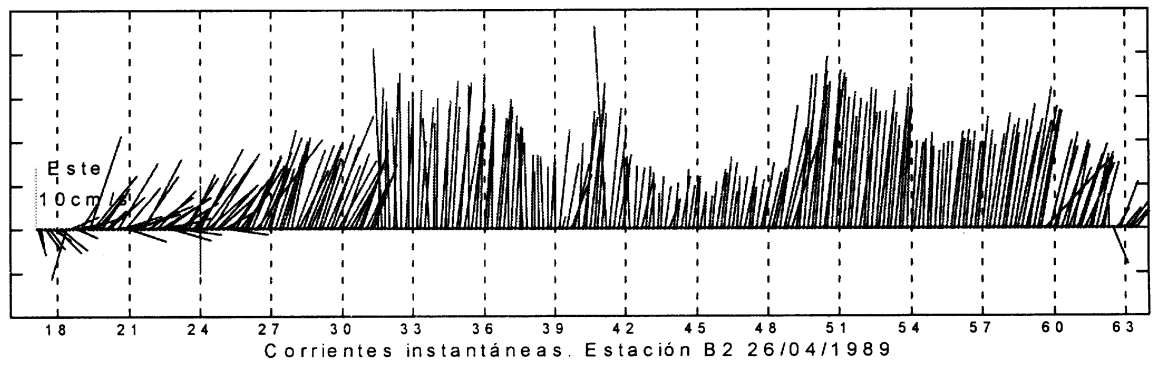

(e)

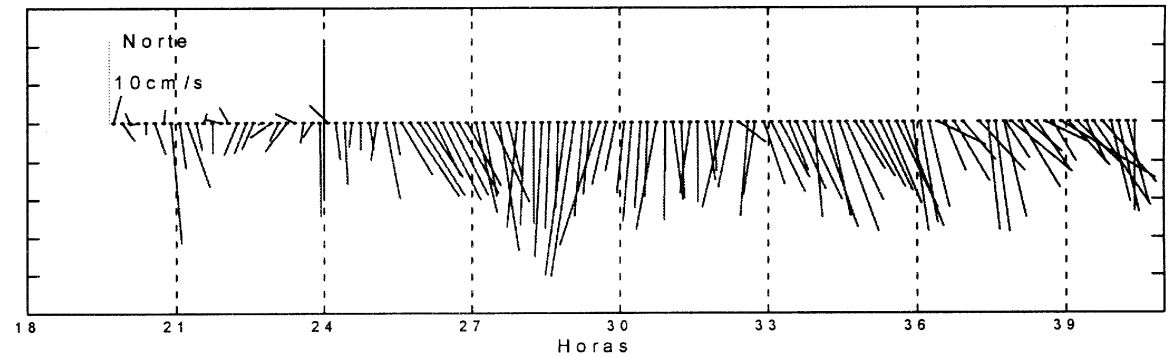

Figura 4. Series de tiempo del vector corriente superficial en formato de astillas, en cada serie el primer trazo denota la escala y la dirección geográfica correspondiente al vector vertical. a) Estación B1, 16/03/1989; b) Estación B1, 25/04/1989; c) Estación B1, 26/05/1989; d) Estación B1, 05/09/1989; y e) Estación B2, 26/04/1989. 
dos por la dinámica impuesta por las mareas semidiurnas. De interés resultan los registros de marzo y septiembre. En el primero, la corriente superficial estuvo dirigida persistentemente hacia el Oeste, es decir aguas superficiales provenientes de la bahía de Bergantín, son inyectadas en la Bahía de Pozuelos. Llama la atención que para el mes de marzo el registro presenta un flujo de drenaje hacia la bahía Pozuelos, que se prolonga por más de $15 \mathrm{~h}$. Para satisfacer la continuidad del flujo, esto parece indicar que debe existir una entrada de agua subsuperficial a Bergantín desde Pozuelos, que no se observa aquí debido a que las mediciones son sólo superficiales, o alternativamente, mientras el flujo sale por el Paso Oeste hacia Pozuelos, agua nueva debe ingresar por el Paso Norte proveniente, ya sea desde Pozuelos o desde la cercana Bahía de Pertigalete. Para septiembre, la corriente superficial en el Paso Oeste se orienta persistente en dirección Este. Es decir, aguas provenientes desde la Bahía de Pozuelos ingresan a Bergantín durante períodos mayores de 46 horas. Este hecho fue reconocido por Senior y Aparicio (5), como producto de un análisis cualitativo. Este tipo de observaciones resulta ser de primordial importancia en el diseño y ejecución de planes de contingencia para minimizar los impactos de posible derrames petroleros, que potencialmente puede producirse en virtud de las operaciones de carga de hidrocarburos pesados y ligeros que rutinariamente se llevan a cabo dentro de la $\mathrm{Ba}$ hía de Bergantín.

\section{Excursión de la corriente superficial}

La excursión de la corriente es una medida indicadora del recorrido espacial que una partícula de agua experimenta bajo la acción de la corriente local. Ella sirve también para tener un conocimiento preliminar de los ejes direccionales en los que el flujo superficial contribuye con mayores desplazamientos.

La Figura 5 muestra la excursión o recorrido de las aguas superficiales. El punto de inicio del trayecto se denota por la coordenada $(0,0)$. Se observa que para el mes de marzo, durante el período de observación no hay indicio de oscilación del patrón de dispersión de una partícula de agua hipotética; sin embargo, es claro que el recorrido del flujo se hace predominantemente según el eje direccional ENE-OSO. Esta persistencia pudiera entenderse como un efecto del forzamiento eólico que se sobrepone al efecto mareal. En términos de afectación, cualquier impacto que se produzca dentro de la bahía de Bergantín, durante este lapso, tendrá como destino la Bahía de Pozuelos.

Para el mes de abril, el patrón de excursión es oscilante con un alcance máximo del orden de $3 \mathrm{~km}$. Es decir que para semejantes condiciones de corrientes una hipotética partícula de agua puede oscilar entre la $\mathrm{Ba}$ hía de Pozuelos y la de Bergantín con una amplitud máxima de $3 \mathrm{~km}$. Es también claro que el eje direccional es similar al observado durante el mes de marzo. Aunque la extensión del registro no permite efectuar el análisis armónico, la forma del recorrido sugiere que, durante el período de observación, la dinámica superficial de la bahía se encuentre controlada por el régimen de marea.

Un patrón algo más complejo se observa en mayo. Allí tenemos dos ejes de flujos: uno según la dirección ESE-ONO con una amplitud de excursión máxima de $1 \mathrm{~km}, \mathrm{y}$ otro según la dirección ESE-OSO y amplitud del orden de $3 \mathrm{~km}$.

Para septiembre, la extensión del registro de observaciones no permite observar periodicidad alguna. Sólo se puede afirmar que el eje direccional de dispersión aparece según la dirección aproximada ESE.

Para la estación B2, el diagrama de dispersión se asemeja en forma y alcance al correspondiente al mes de mayo en la estación B1. Aun cuando estos registros correspondan a fechas diferentes, resulta interesante notar la consistencia visual entre ellos. Ello pudiera indicar la existencia de condiciones donde la entrada de agua por el paso norte 
(a)

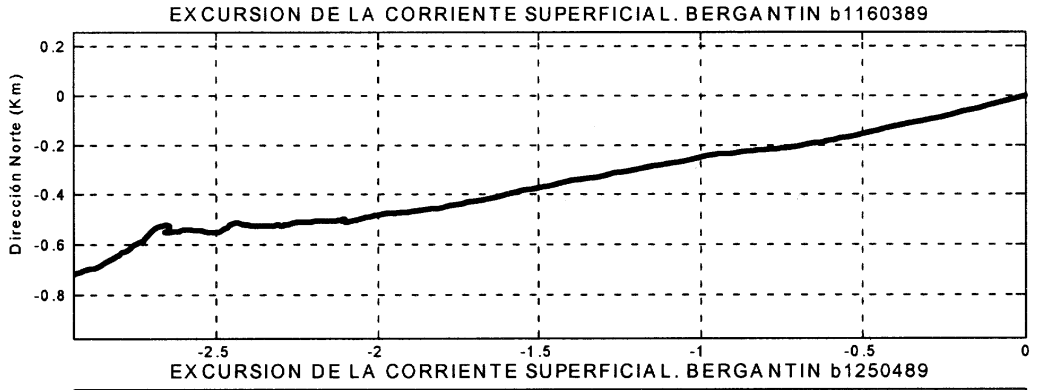

(b)

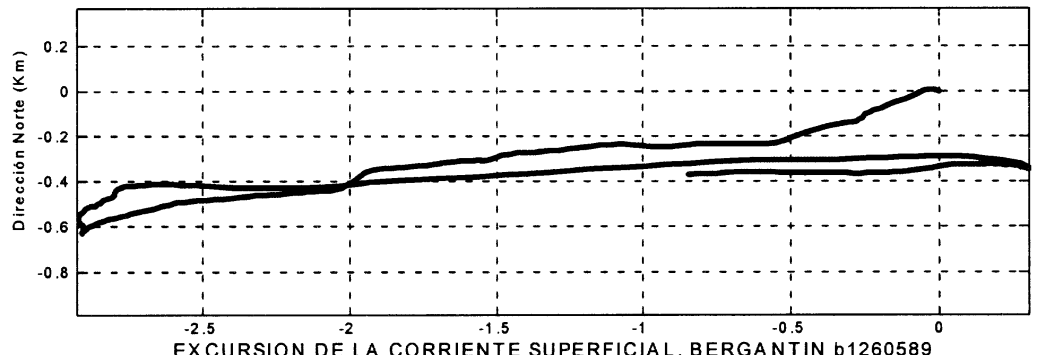

(c)

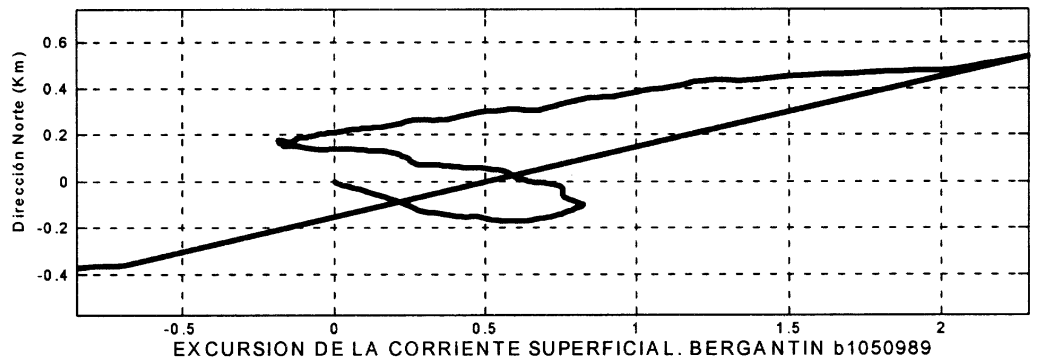

(d)

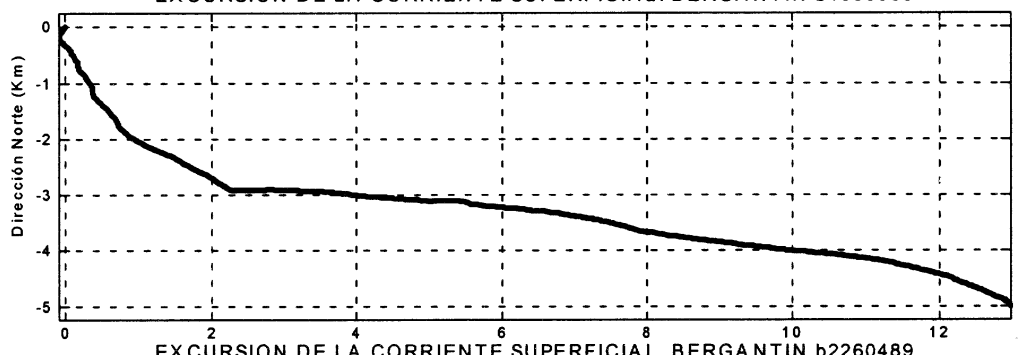

(e)

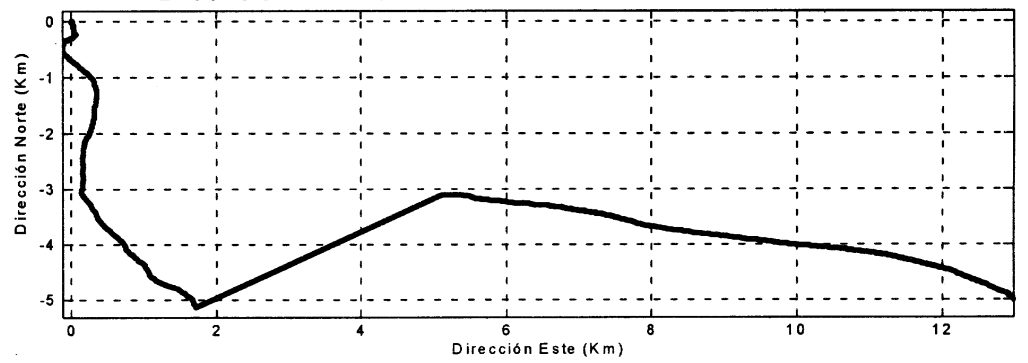

Figura 5. Excursión o recorrido espacial, en $\mathrm{km}$, de la corriente superficial en algunos días de diferentes meses. El punto (0,0) representa el inicio temporal del recorrido.a) Estación B1, 16/03/1989; b) Estación B1, 25/04/1989; c) Estación B1, 26/05/1989; d) Estación B1, 05/09/1989; y e) Estación B2, 26/04/1989. 

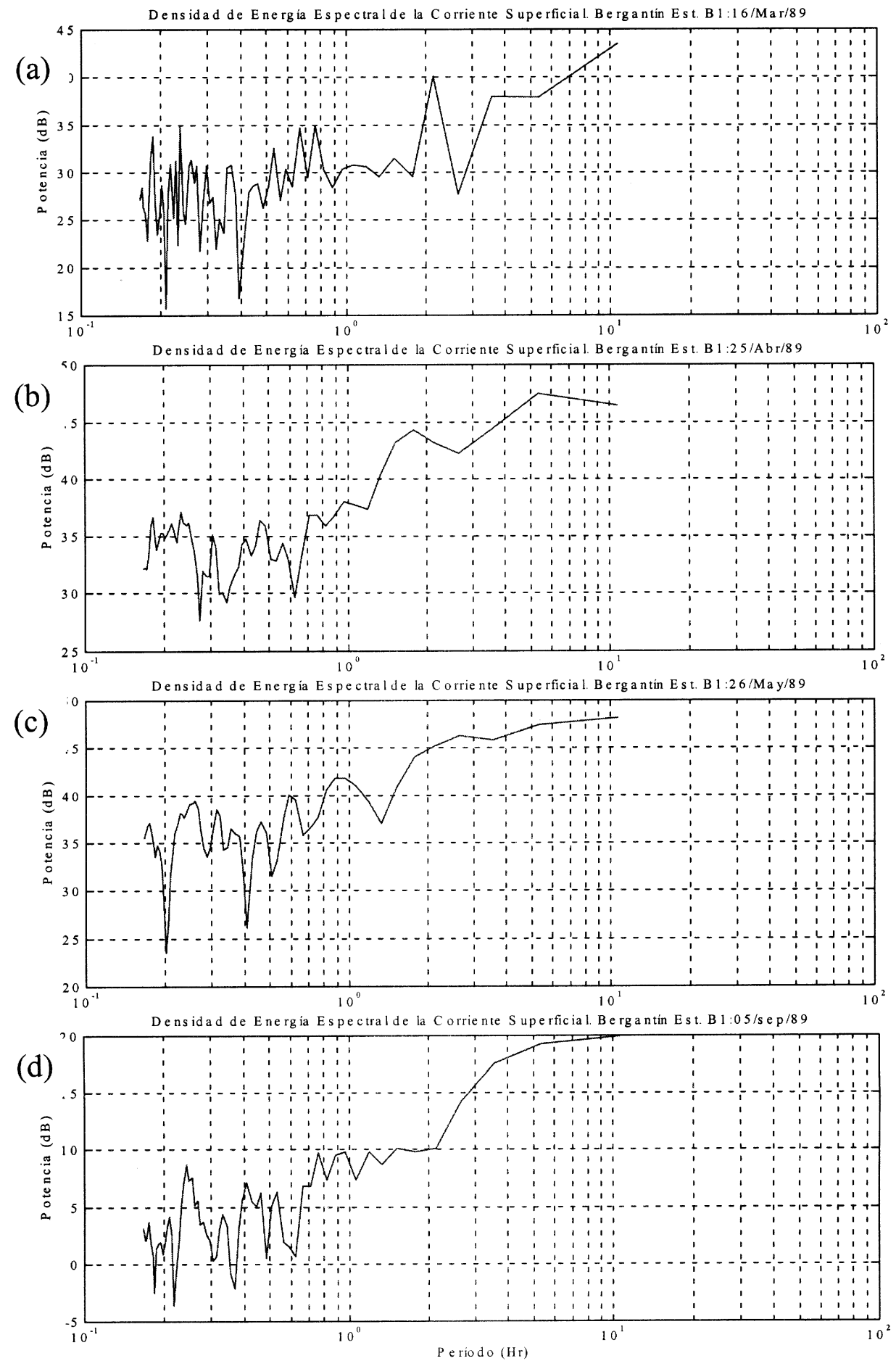

Figura 6. Densidad de energía espectral de la magnitud de la corriente superficial aplicada a las mediciones de corrientes superficiales en la estación B1.a) 16/03/1989; b) 25/04/1989; c) $26 / 05 / 1989 ;$ y d) $05 / 09 / 1989$. 
de Bergantín está en fase con la salida por el paso oriental. Lo cual tendría un efecto de arrastre muy fuerte sobre las aguas residentes en la Bahía de Bergantín, las cuales podrían renovarse en escalas temporales asociadas a los ciclos de mareas dominantes (6-12 h), es decir que durante esos períodos, el tiempo de residencia de partículas en la superficie sería relativamente corto. Sin embargo, bajo las condiciones de vientos débiles el destino de cualquier contaminante superficial estará bajo los efectos oscilantes de la marea. Ello puede entenderse que durante esos períodos un eventual derrame de crudo que ocurra dentro de la Bahía de Bergantín y que en primera instancia se dirija hacia la Bahía de Pozuelos, al producirse la reversión del flujo, aquella porción de la mancha de petróleo que este dentro del radio de $3 \mathrm{~km}$ del Paso Oeste de Bergantín, tiene dinámicamente la posibilidad de re-ingresar la Bahía de Bergantín.

\section{Densidad espectral de la magnitud de la corriente}

La señal registrada por un correntímetro contiene información de todos los actores físicos que de una manera $u$ otra pueden inducir movimiento. En agua costeras es bien conocido el efecto que tiene los desplazamientos verticales del agua asociados a las mareas sobre las corrientes horizontales. De igual manera el viento induce movimientos a través de la entrada de momentum en la superficie, el cual a su vez se propaga dentro de la columna de agua e induciendo en última instancia movimientos horizontales y verticales. El agua superficial se ve afectada directamente por el viento en escalas espaciales y temporales del orden $1 \mathrm{~km}$ y $1 \mathrm{~h}$ respectivamente. Para escalas mayores aparecen otras contribuciones, tales como las atribuidas al efecto de Ekman. La presencia de gradientes horizontales de densidad (tales como los encontrados en las cercanías de las desembocaduras de los ríos), es también un factor importante en la generación de movimientos horizontales.
En vista que se disponía de un mejor conjunto de datos para la estación B1, ésta se escogió para hacer la descomposición espectral de la magnitud de la corriente. El eje horizontal representa cada uno de los posibles períodos que se pueden extraer de la señal disponible. Es decir, si la duración de las mediciones es de $13 \mathrm{~h}$ sólo se podrán buscar señales en el registro con períodos menores a $13 \mathrm{~h}$. El eje vertical representa la potencia que existe en cada uno de esos períodos.

En general se puede observar que la curva que describe la energía por períodos es análoga en todos los meses (Figura 6). Nótese la presencia de dos picos con períodos de 2 y $12 \mathrm{~h}$ en los registros de marzo y abril, con energías del orden de 40-45 db y 45-47 $\mathrm{db}$, respectivamente. En ellos el pico con período semidiurno $(12 \mathrm{~h})$ podría atribuirse al efecto combinado viento/mareas. Sin embargo, el pico asociado a una señal con período de $2 \mathrm{~h}$ no pudo asociarse a los agentes físico conocidos. Se presume, que podría ser el resultado de los subarmónicos de las mareas, que se producen por la fricción y deformación que sufre la onda de marea cuando ingresa a aguas costeras (2), otra alternativa es que sean frecuencias espurias creadas por el método de Welch. No obstante, dado el hecho de que esta frecuencia (período) esté dentro del límite teórico de frecuencias resolubles dado por $f_{0} \leq f \leq f_{N} ;$ donde $f_{N}=1 / 2 \Delta t$ es la frequencia de Nyquist y $f_{O}=1 / N \Delta t$ es la frecuencia más baja que el análisis espectral puede resolver, se cree que esta señal esta asociada a algún agente físico externo.

Para las gráficas restantes de Mayo y septiembre la energía contenida en los períodos de $1 \mathrm{~h}$ es relativamente inferior a las primeras. Sin embargo, el espectro posee más energía hacia los períodos típicos de la marea.

\section{Discusión y Conclusiones}

Se muestra que a pesar de las limitaciones en cuanto a la continuidad y simultaneidad temporal de las observaciones direc- 
tas sobre las que se apoya este trabajo, las corrientes superficiales en la Bahía de Bergantín exhiben un alto grado de complejidad en respuesta a una interacción entre los campos de vientos y mareas y su interacción con la topografía local.

Se muestra que, espectralmente la banda de frecuencia asociada a vientos del $\mathrm{NE}$ con períodos de 6,12 y $24 \mathrm{~h}$ es el agente con mayor potencial inductor de corrientes superficiales. La coincidencia de esas bandas energéticas con la de los constituyentes cuarto, semidiurnos y diurnos de la marea, podría representar un factor de alto riesgo para las operaciones de carga de hidrocarburos dentro de la bahía, pues la conjunción del viento con un estado de marea favorable amplificaría el transporte y por ende la dispersión de cualquier mancha de hidrocarburos. El riesgo es mayor durante el mes de septiembre, cuando se produce la reversión del campo de vientos (vientos del SurOeste), dado que existe la posibilidad de ocurrir eventos de resonancia entre una marea llenante y esos vientos concurrentes.

Estas afirmaciones no son conclusivas y deben sustentarse con mediciones sinópticas de corrientes de mayor cobertura espacial y temporal. En particular, sería de mucho interés desarrollar una modelo hidródinamico de corrientes de mareas y usarlo para motorizar un modelo de dispersión de hidrocarburos.

$\mathrm{El}$ análisis de las observaciones de corrientes superficiales demuestra la necesidad de establecer un programa de registro local de carácter permanente de la variable intercambio de agua entre la Bahía de Bergantín y sus áreas adyacentes (Bahías de Pozuelos y Pertigalete), basado en el monitoreo sinóptico de las parámetros físicos más relevantes: vientos y corrientes. Sólo así podría reforzarse criterios técnicos para responder efectivamente a la crucial interrogante del destino final de posibles contaminantes presentes tanto en la Bahía de Bergantín como en sus zonas inmediatas. Sería de mucho interés el desarrollo de modelos numéricos para reproducir la hidrodinámica y estudiar los procesos de dispersión en toda la zona y entender así el balance de masa y elementos contaminantes entre las bahías.

\section{Agradecimientos}

Los autores de este trabajo agradecen al Convenio UDO-CORPOVEN, el auspicio y financiamiento de esta investigación, así como a Manuel Gil su valiosa ayuda durante el trabajo de campo para la captación de las mediciones. A la Guardia Nacional en el Puesto de Vigilancia Costera de Punta de Meta, por el apoyo en la logística de campo. Por último y no menos, se agradecen los comentarios y sugerencias de uno de los árbitros los cuales ayudaron a clarificar algunos aspectos de este trabajo.

\section{Referencias Bibliográficas}

1. KRAUSS T.P., SHURE L., LITTLE J.N. Sig nal Processing Toolbox User's Guide, Mathwork Inc. Maas. 01760-1500, pp 240, 1994.

2. OKUDA T. Bolt Inst Oceanogr Univ Oriente 20:1-2, 1981.

3. GODIN G. The Analisis of Tides, University of Toronto Press (Canada), pp. 264, 1972.

4. APARICIO R. Intercambio de agua superficial entre la Bahía de Bergantín y regiones adyacentes, Estado Anzoátegui, Venezuela. Implicaciones en la problemática local de dispersión de contaminantes. Resúmenes del IV Congreso Interamericano sobre el Medio Ambiente, USB-CONICIT, Caracas (Venezuela), pp. 320, 1997.

5. SENIOR W., APARICIO R. Estudio Ambiental de las Costas del Estado Anzoátegui (Proyecto EACA). Bahía de Bergantín (Informe Técnico), Protección Integral Corpoven-Instituto Oceanográfico de Venezuela, pp. 56, 1992. 